\title{
STUDY OF THERMAL CREEP OF COATED CLADDING MATERIALS
}

\author{
Martin ŠEvečeK ${ }^{a, b, *}$, Koroush ShiRvan $^{b}$, Ronald G. Ballinger $^{b}$ \\ ${ }^{a}$ Department of Nuclear Reactors, Faculty of Nuclear Sciences and Physical Engineering, Czech Technical \\ University in Prague, V Holešovičkách 2, Prague 8, Czech Republic \\ ${ }^{b}$ Department of Nuclear Science and Engineering, Massachusetts Institute of Technology, 77 Massachusetts Ave, \\ Cambridge, MA, USA \\ * corresponding author: martin.sevecek@fjfi.cvut.cz
}

ABSTRACT. Coated cladding materials are considered as a near-term concept for so-called Accident Tolerant Fuel materials. Their behavior in accidental conditions is mainly studied around the world, however, they need to survive in-reactor normal operation before reaching theoretical accidental conditions. An out-of-pile experiment was designed to study accelerated inward thermal creep and outward displacement of a cladding that occurs after pellet-cladding interaction. Four different materials deposited by the cold-spray technique were studied as well as the reference uncoated one. The results confirm theoretical prediction that due to a mismatch between the fundamental physical properties of each layer, high stresses will build up and the plastic strains expected will lead to coating cracking.

Keywords: Accident Tolerant Fuel, Fuel Cladding, Creep, Coating.

\section{INTRODUCTION}

Zirconium alloys have been used as a fuel cladding by the nuclear industry for decades in Light Water Reactors (LWRs) as well as other reactor designs such as CANDU or RBMK. Despite the widespread use of zirconium alloys in nuclear applications, relatively little creep data exists for these materials.

Creep is slow time- and temperature-dependent strain that occurs in a material under load at higher temperatures. In a creep test, a constant load is applied to a specimen maintained at a constant temperature. The strain is then measured over a period of time. Primary creep is a period of primarily transient creep with decreasing creep rate due to work hardening of the material. Then secondary creep is a period with roughly constant creep or strain rate due to which is also referred as steady-state creep due to balance between work hardening and annealing. If the cross-sectional area reduces enough, tertiary creep occurs. The creep rate increases due to necking and increased local stress and eventually results in fracture as shown in Fig. 1. Irradiation creep is the additional strain that occurs as a result of irradiation and stress in material that needs to be considered in nuclear applications [1. The presented experiment was performed ouf-of-pile therefore only thermal creep was studied.

At the beginning of the nominal LWR operation, the cladding is subjected to the differential pressure (coolant pressure minus internal gas pressure) and creeps down in compression due to this loading [3]. Progressively, the pellet-cladding gap closes under the combined effect of the cladding creep and the pellet swelling induced by fission product accumulation.

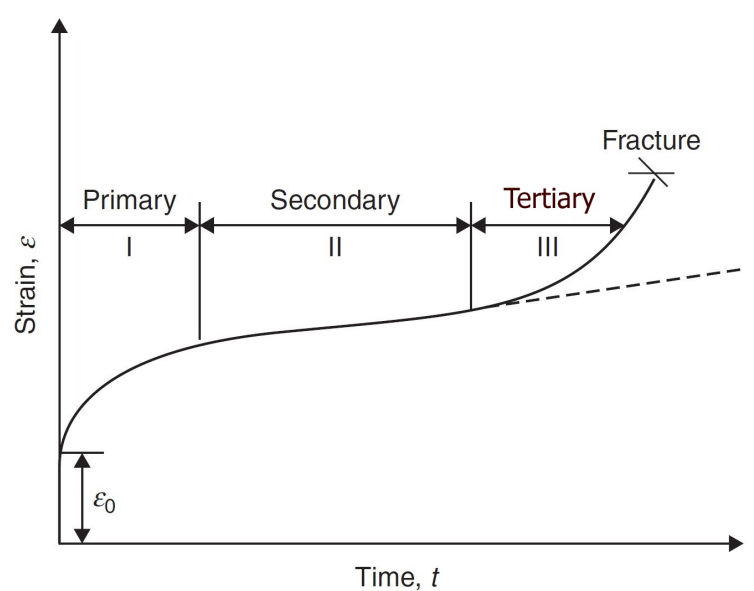

Figure 1. Typical creep curve for materials under load at elevated temperature [2].

While the gap closes, the cladding, initially loaded in compression, progressively gets in contact with the pellets and becomes loaded in tension 4 . The tensile stress increases slowly up to about 20 to $30 \mathrm{MPa}$, which corresponds to an equilibrium value, according to the cladding creep rate and the pellet swelling rate. [5, 6]

An experimental setup was designed and built to simulate this in-reactor behavior of cladding materials out-of-pile. The nuclear fuel cladding can be loaded in compression or tension between $0-350 \mathrm{MPa}$ at the temperature between $20-320^{\circ} \mathrm{C}$. The samples were partially instrumented and some of the parameters were evaluated outside of the autoclave after a certain testing period. 


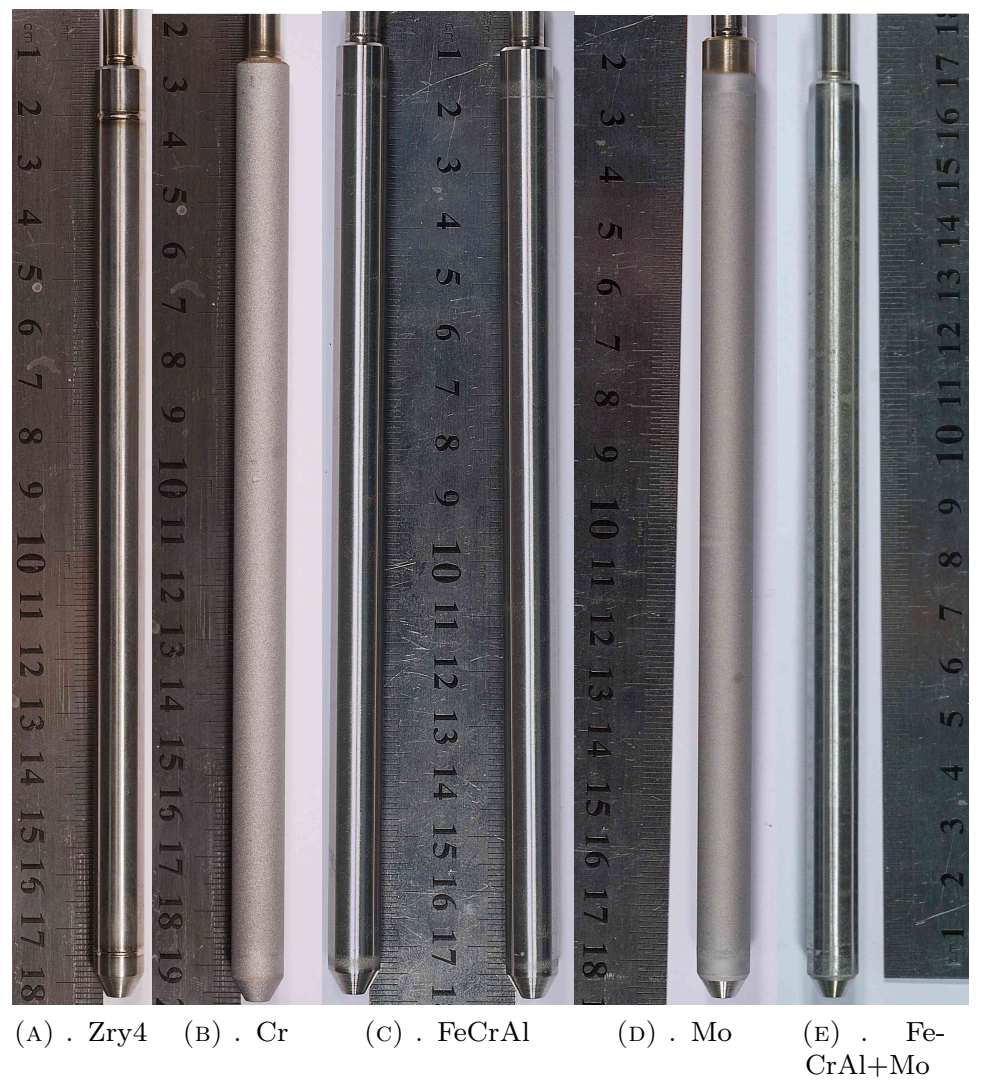

Figure 2. Pressure tubes for creep experiments in as-coated state. Cr-coated tube is fully coated including the welds and the end cap, the other coatings cover linear part of tubes only.

The main objective of this experiment is to study so-called Accident Tolerant Fuels [7. There are many concepts of ATF under development all around the world and one of the most promising near-term design is the multicomponent cladding [8-11]. Zr alloys still serve as the main structural component on which protective coatings are applied. These coatings have slightly different physical behavior than the substrate (e.g. thermal expansion, swelling) which induces high stresses into the cladding and results in high plastic strains [12]. Additionally, the coatings impose high stresses originating from the particular deposition process used. There is a concern that the coating or cladding might be disrupted when these loads are combined with other in-reactor strains such as creep described above. Therefore, the presented experiment emulates the strains typical of in-reactor conditions with advanced cladding materials and its response is studied.

\section{MATERIAls AND Methods}

Standard commercially available Zircaloy-4 was used as a substrate for all tests. The composition is as follows: $1.32-\mathrm{Sn}$; 0.21-Fe; 0.11-Cr; 0.13-O; Bal-Zr. The substrate was surface polished in ethanol lubricant with 320 grit $\mathrm{SiC}$ abrasive paper before deposition of coatings, degreased and cleaned in DI water, ethanol and acetone in agreement with [13. Four different coating materials were deposited by the cold spray technique - pure Cr (deposited by Army Labs), Fe$\mathrm{CrAl}$ - low alloy (LA); pure $\mathrm{Mo}$; and $\mathrm{FeCrAl}+\mathrm{Mo}$ multilayer (deposited by University of WisconsinMadison). The as-coated tubes are shown in Fig. 2 Uncoated Zircaloy-4 was also studied as the reference case. The reference uncoated Zry-4 pressure tube and the as-coated samples are shown in Fig. 2

The standard Zry-4 tubes were welded with an end cap on one end and with a thinner Zry-4 tube at the other end. The as-prepared samples were then pressurized by air at room temperature up to $30 \mathrm{MPa}$ to check the quality of welds. The Zry-4 samples were then coated using the cold spray technique with pure Chromium, Molybdenum, FeCrAl low alloy and multilayer of $\mathrm{FeCrAl}+\mathrm{Mo}$. The leakage test was then repeated using air. Samples were pressurized by air at room temperature up to $11 \mathrm{MPa}$ for 5 minutes with no pressure decrease. Then He leakage test was performed with the base pressure of 0.013332 $\mathrm{Pa}$ with the leak rate $1.3 \mathrm{E}-8 \mathrm{~Pa}$ *liter/second and no indications. There was a concern that the welding can be partially disrupted by cold spraying but it was not confirmed. A detail of the over-coated weld with $\mathrm{Cr}$ is shown in Fig. 3

Two FeCrAl LA coated tubes (PT-12 and PT-13) were tested. The tubes have been polished after deposition with 600 grit $\mathrm{SiC}$ paper and the surface roughness 


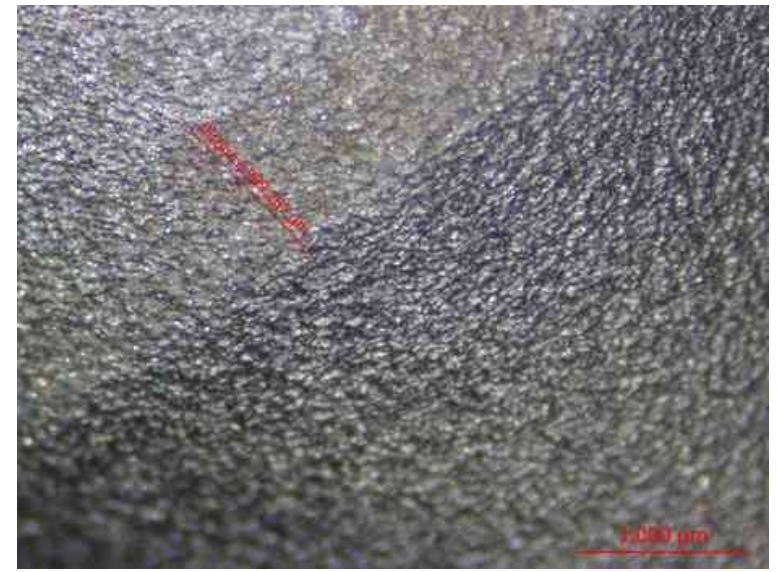

Figure 3. Cr CS coated pressure tube - detail of the bottom weld.

is in the range 250 to $320 \mathrm{~nm}$. The thickness of the coatings is $0.097 \pm 0.006 \mu \mathrm{m}$. The FeCrAl low alloy composition in weight percentage is Fe-10Cr-6Al. Two pressure tubes identified as PT-17 and PT-18 were coated with Mo and then over-coated with $\mathrm{FeCrAl}$ LA. The linear length of the tubes is coated but the curved end caps are not coated. Because the surface roughness plays a role in mechanical and corrosion behavior, the entire sample surface was polished with 600 grit $\mathrm{SiC}$ paper. The surface roughness is in the range of 250 to $360 \mathrm{~nm}$ and the average thickness of the coating system is $110 \pm 10 \mu \mathrm{m}$. Some roughness remained after polishing on both samples. Similar to the other $\mathrm{Mo} / \mathrm{FeCrAl}$ samples, uniform deposition of the FeCrAl LA on top of the as-deposited Mo was difficult. This resulted in some thinner regions of FeCrAl. To ensure that the FeCrAl coating is not breached, which would expose Mo inter-layer or Zr substrate, polishing was stopped wherever FeCrAl layer was less than or equal to $50 \mu \mathrm{m}$. Two PTs (PT-15 and PT-16) were coated with Mo. Only the linear length of the tube is coated and curved end caps are not coated. The tubes have not been polished as it might breach the coating due to its minimal thickness. The thickness of the coatings is about $27 \mu \mathrm{m}$ as measured by a micrometer (including surface roughness). Pure molybdenum was deposited on the substrate as confirmed by XRD after deposition. The X-ray diffraction pattern of the Mo coating (not shown here) showed perfect agreement with Mo reference with no extraneous peaks originating from oxides or other impurities. Pressure tubes coated with $\mathrm{Cr}$ were prepared using VRC Gen III system at ARL, MA. The detailed deposition parameters can be found in 8 .

\subsection{Cold Spray Technique}

All tested samples were coated using the cold spray process (CS). It involves the acceleration of micronsized particles in the form of powders that are carried in a high pressure and sometimes heated gas stream in the solid state toward a suitable substrate. During the

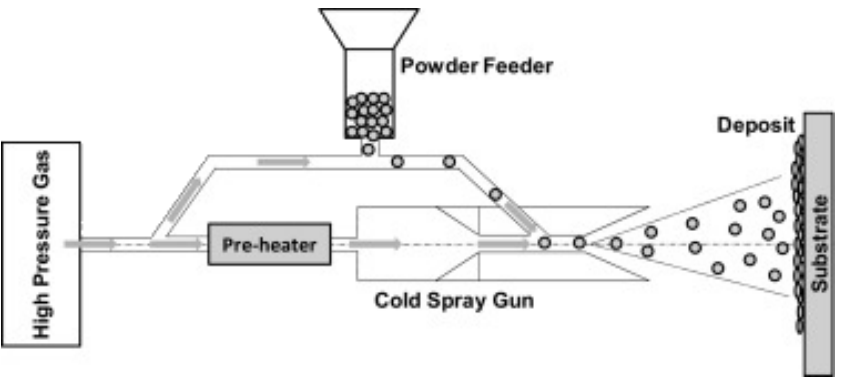

FigURE 4. Schematic representation of cold spray process 14.

interaction with the substrate, the particle undergoes tremendous plastic deformation. Upon impact, the plastic deformation disrupts and breaks down surface oxide layers on both the powder and substrate leading to a metallurgical bond, as well as mechanical interlocking. Since the feedstock powder is deposited in the solid state, the microstructure is retained after deposition with the exception of dynamic recrystallization due to high strain levels. A schematic representation of the CS is shown in Fig. 4

\section{Experimental Setup}

The simplified schematics of the experimental setup is shown in Fig. 5. Two independent heaters are placed onto the autoclave designed for pressure up to 24.13 MPa (3500 psi) and thermally insulated from outside. They are controlled by the in-house designed controller that uses two thermocouples and two pressure gauges. Four proportional-integral-derivative (PID) controllers are interconnected to ensure that the parameters are within allowable limits and define the temperature of the preheater and autoclave by providing power to heaters. The water pressure is controlled by high-pressure pump which will run throughout each experiment on one side and by the back pressure regulator on the other side. The autoclave pressure for the experiments was between 13.4 - 14 MPa depending on the particular test. Instrument leads as well as the pressure tubes exit the autoclave through an appropriately configured Conax fittings. A data acquisition system records temperature from four thermocouples. Two are inserted into the autoclave at different axial positions, one measures and controls the preheater and the last checks the temperature at the exit of the high-pressure part of the loop for safety reasons. The system collects also pressure from three pressure transducers. One is installed at the high-pressure pump outlet and controls the high pressure inside the autoclave, one is connected to the tubing on the low-pressure section of the water loop and the third is connected to the inert gas inlet and controls the Ar pressure inside cladding tubes. Omega pressure gauge and Omega DP41-E unit were used to measure argon pressure inside the gas line.

Agilent 34970 A was used as a data acquisition unit and also as an $\mathrm{AD}$ converter. The data measured were 


\begin{tabular}{ll}
\hline Coolant & Pressurized H2O \\
\hline Temperature $\left[{ }^{\circ} \mathrm{C}\right]$ & $280-320$ \\
\hline System pressure $[\mathrm{MPa}]$ & $12.4-14.47$ \\
\hline Tube internal pressure $[\mathrm{MPa}]$ & $0.1-41.0$ \\
\hline Coolant pH $\left(\right.$ at $\left.25^{\circ} \mathrm{C}\right)$ & $6.6-7.3$ \\
\hline Inlet conductivity $[\mathrm{mS} / \mathrm{cm}]$ & $0.01-0.05$ \\
\hline Outlet conductivity $[\mathrm{mS} / \mathrm{cm}]$ & $0.03-0.1$ \\
\hline Ox. concentration $[\mathrm{sat} \%]$ & $0.39-0.4$ \\
\hline
\end{tabular}

TABLE 1. Experimental parameters for autoclave coolant.

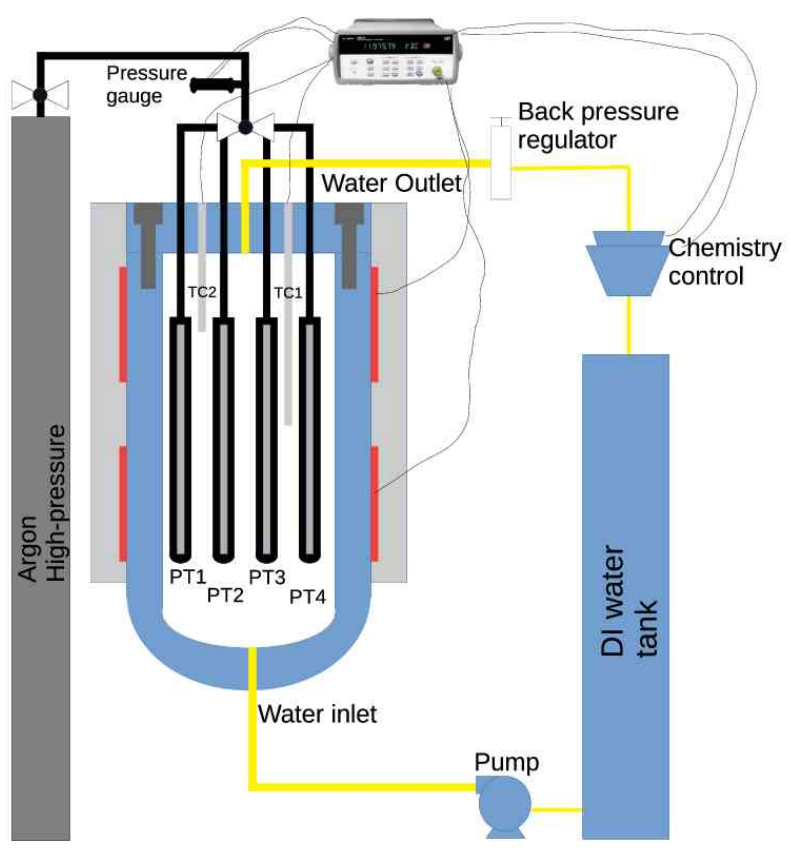

Figure 5. Schematics of the experimental setup.

then stored in a computer. Thornton Multiparameter 770MAX unit was used to collect inlet and outlet water conductivity, $\mathrm{pH}$ and oxygen concentration. Matheson hydrogen generator NM Series II was used to generate hydrogen and bubble it into the DI water tank where the partial pressure of the gases was measured.

Water conductivity meters, $\mathrm{pH}$ meter, and oxygen meter were used to control the chemistry inside the system. Additionally, hydrogen was bubbled into the water tank to reduce corrosion by reducing electrochemical potential. Hydrogen concentration was not measured but it can be calculated from the partial hydrogen pressure inside the water tank using Henry's law considering that the DI water inside the tank is saturated. The gas line interconnecting the pressure tubes with Ar gas tank was first under atmospheric pressure which results in the downward creep of the cladding (compression) due to the differential pressure inside the autoclave. Later the gas line was pressurized by argon up to $41.36 \mathrm{MPa}$ (6000 psi) to simulate the outward creep (tension).

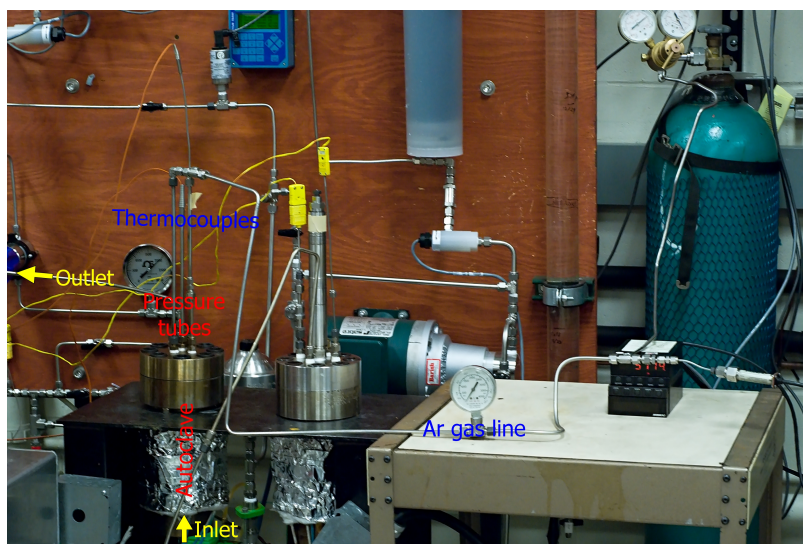

Figure 6. Photo of the autoclaves, gas system and water loop.

The picture of the experimental setup is shown in Fig. 6. The second autoclave can be interconnected and allows to test more samples at the same time. The autoclaves are dynamic and allow online control of the chemistry as well as purification of the DI water. Small leakages were found in the gas line that pressurizes pressure samples during the test e.g. in Fig. 12 (green line). For that reason, the load was slowly decreasing. Later the tubing was replaced and lower loads were applied with no leakages.

\section{Results}

Due to the limited length of this paper, only results for FeCrAl+Mo samples (PT-17, PT-18) are shown. Three consequent test runs were performed. During the first run, the pressure tubes were under atmospheric pressure and the autoclave pressure was around 13.5 MPa resulting in fast down creep. Because the outer surface of the pressure tubes is exposed to the standard HWC environment [15] the pressure tube corrodes. For that reason, the chemistry of the environment was monitored. The temperature of the system was between $305-315{ }^{\circ} \mathrm{C}$ and hydrogen was bubbled into the DI water tank to reduce oxygen concentration and the electrochemical potential. The oxygen concentration during Run 1 is shown in Fig. 9. The fluctuations are caused by periodic operation of the hydrogen bubbler but the concentrations are generally very low. Water $\mathrm{pH}$ slowly decreases during all runs between 7.2 down to 6.7 at the end of the test. The water conductivity was measured at the inlet and outlet of the system. A filter is placed between them to clean the water and keep its properties during the test. The rapid increase of the outlet conductivity at the beginning of the test is probably caused by rapid corrosion of Mo. Mo quickly corrodes and creates volatile oxide in HWC environment. Mo should be fully overcoated by FeCrAl but it seems that some parts have defected or some residual Mo was left on the outer surface. The corrosion reduced after about 20 hours as shown in Fig. 8. 


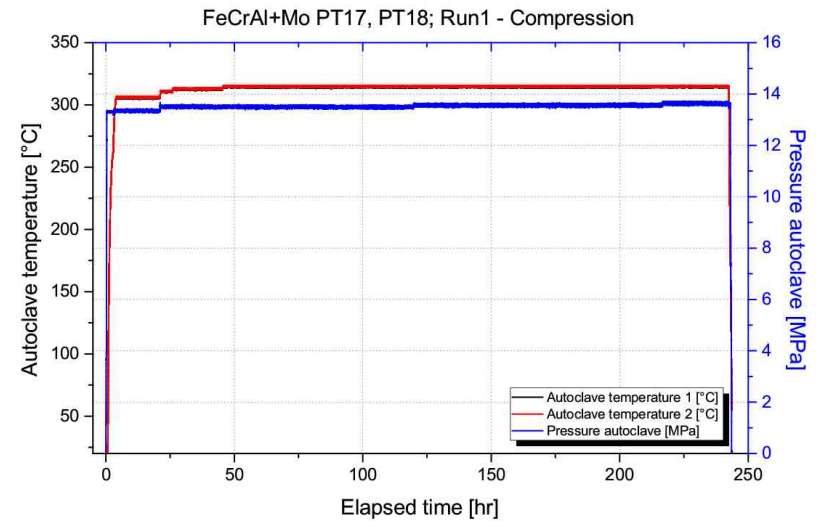

Figure 7. FeCrAl+Mo Run 1 - autoclave temperature and pressure.

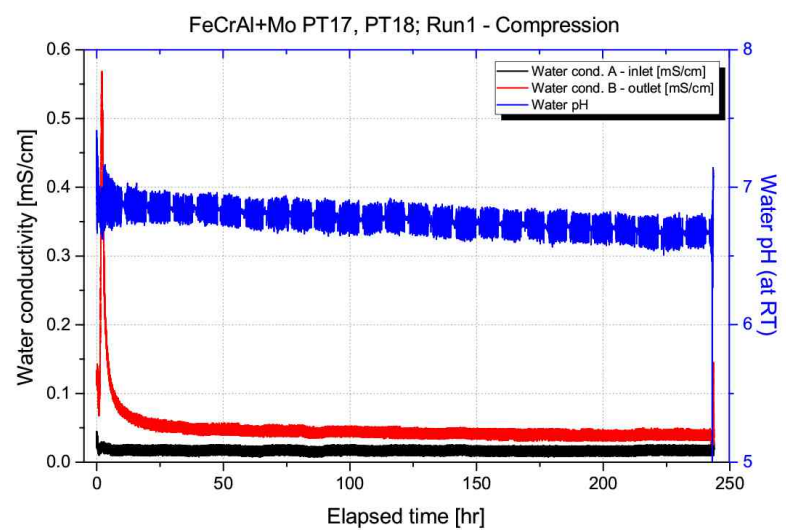

Figure 8. FeCrAl+Mo Run 1 - Water inlet and outlet conductivity and $\mathrm{pH}$.

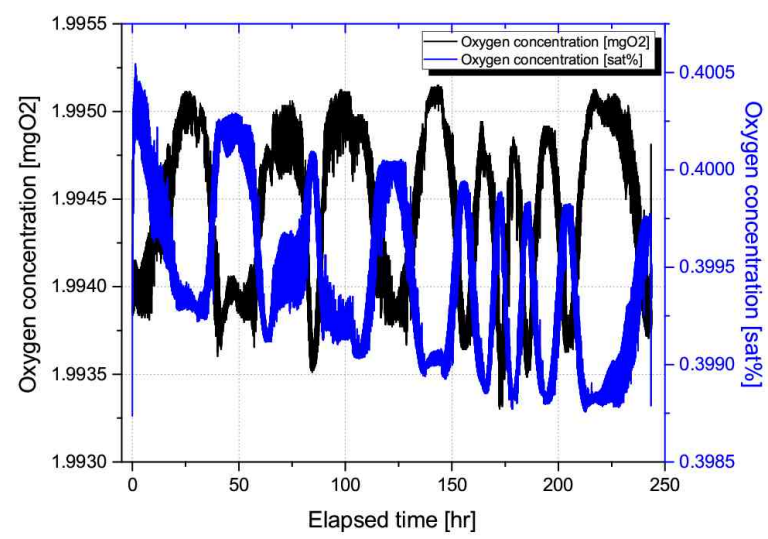

Figure 9. FeCrAl+Mo Run 1 - oxygen concentration.

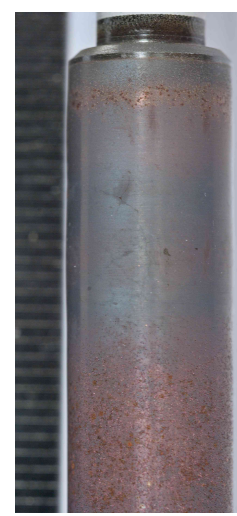

(A) . Top

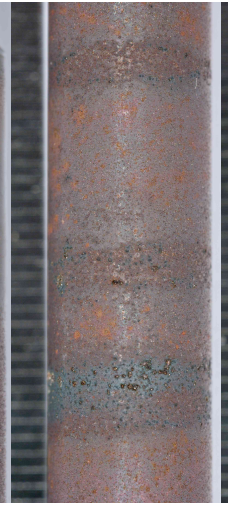

(B). Overcoat

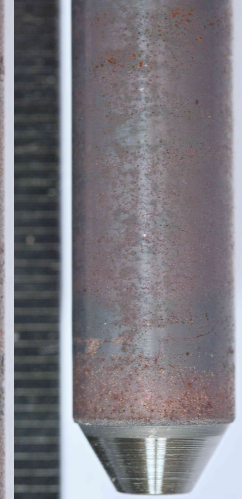

(c) . Bottom
Figure 10. Visual inspection of $\mathrm{FeCrAl+Mo} \mathrm{pressure}$ tubes after Run 1 - compression.

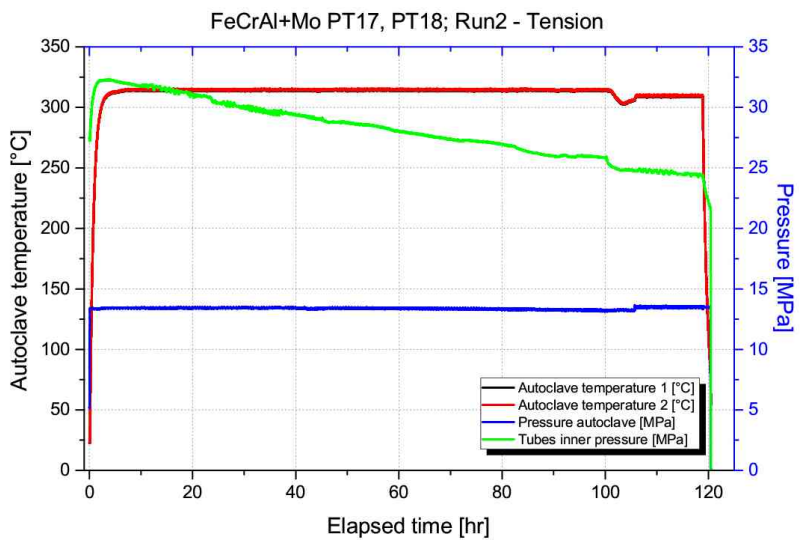

Figure 11. FeCrAl+Mo Run 2 - autoclave temperature and pressure, inner tube pressure.

The Run 1 when pressure tubes were under compression lasted 245 hours. The system was then cooled and depressurized and samples visually examined. The samples were then placed back into the autoclave and Run 2 was initiated. The tubes were first pressurized by Ar from the gas line and the system was filled with water and pressurized. After cleaning of the loop the heatup of the autoclave started. The leakage of $\mathrm{Ar}$ in the gas line was discovered but the test continued for 120 hours. As can be seen from Fig. 12 the water conductivity increased rapidly at the beginning after loading the samples with the inner load. The decrease of conductivity is much slower in comparison with Run 1 which suggests that the $\mathrm{FeCrAl}$ coating cracked due to excessive loads and exposed Mo coating. Run 2 was interrupted due to leakages in the gas line. Samples were visually inspected and then placed back for Run 3 for another 190 hours.

The leakage of Ar was repaired so that the load can be constant during the run. The inner pressure reached $28 \mathrm{MPa}$. The autoclave temperature and pressure were similar to previous runs. As shown in Fig.16. there was a sudden increase of water conductivity after 140 hours. It was explained by a crack in the 


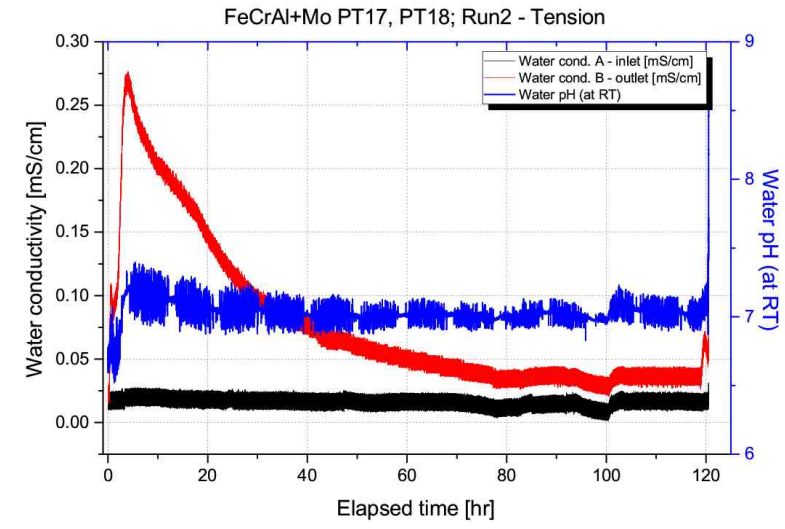

Figure 12. FeCrAl+Mo Run 2 - Water inlet and outlet conductivity and $\mathrm{pH}$.

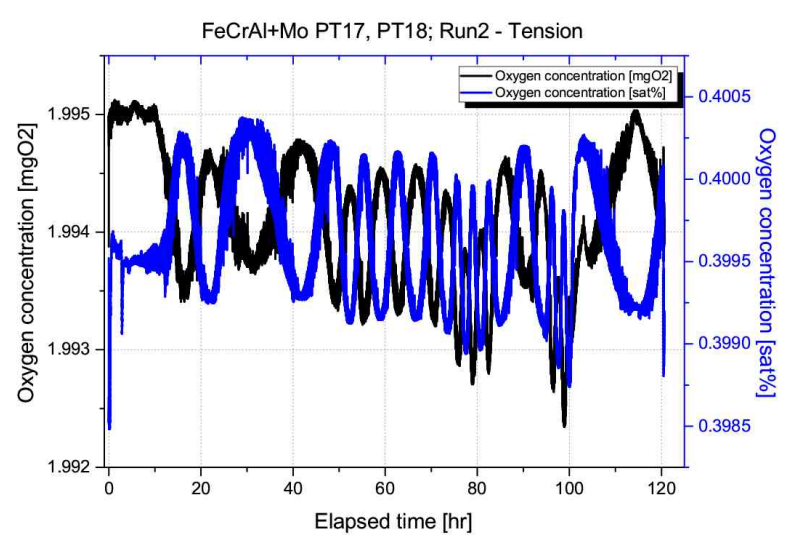

Figure 13. FeCrAl+Mo Run 2 - oxygen concentration.

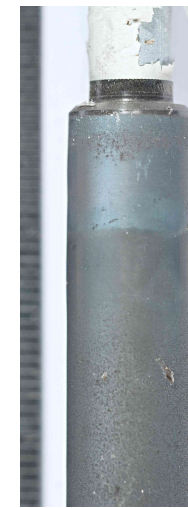

(A) . Top

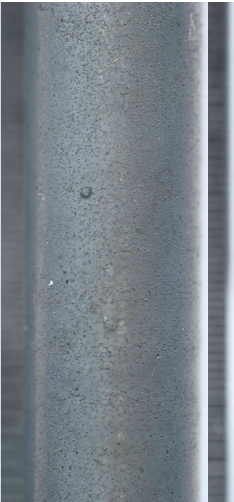

(в) . Overcoat

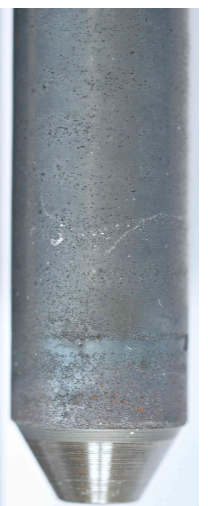

(c) . Bottom
Figure 14. Visual inspection of $\mathrm{FeCrAl}+$ Mo pressure tubes after Run 1 and Run 2 - tension.

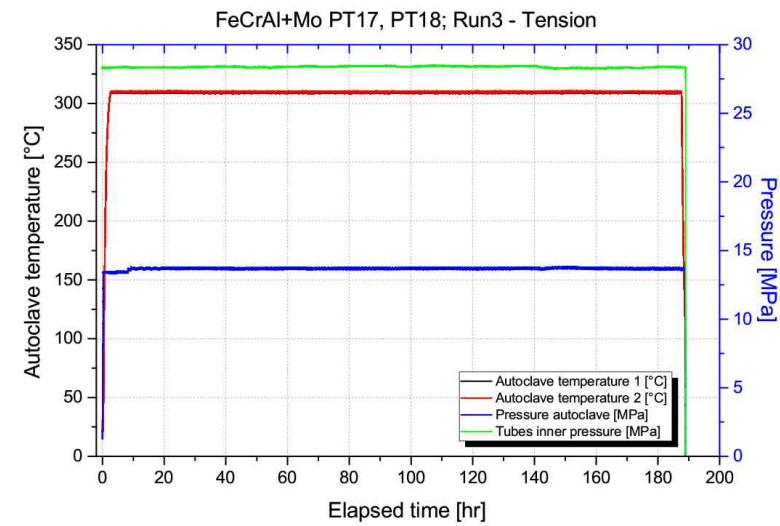

Figure 15. FeCrAl+Mo Run 3 - autoclave temperature and pressure, inner tube pressure.

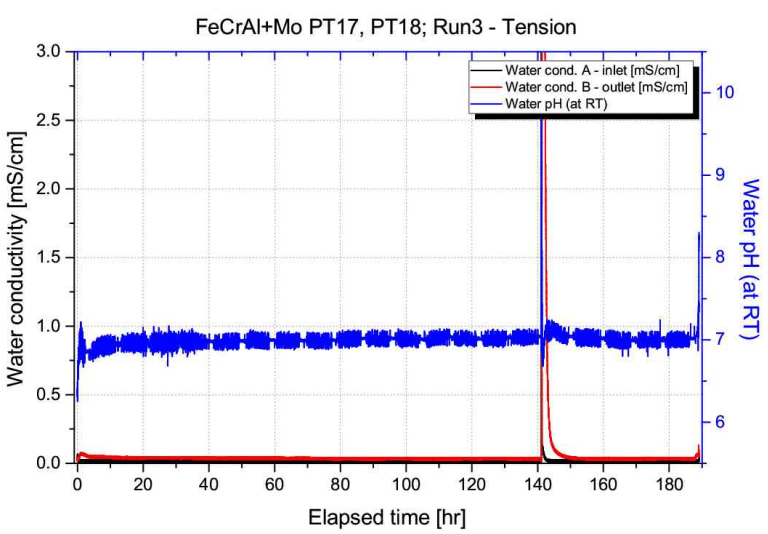

Figure 16. FeCrAl+Mo Run 3 - Water inlet and outlet conductivity and $\mathrm{pH}$.

coating on PT-18 that was later identified. This crack was much bigger in comparison with the micro-cracks observable under a microscope. Visual inspection of the samples showed different axial corrosion behavior. Most of the sections of the samples formed dark red iron oxide similar to FeCrAl PT-12 and PT-13 where no interlayer was applied. However, some parts of the sample were dark grey and black. It seems that the $\mathrm{FeCrAl}$ did not fully protect Mo inter-layer in these regions. Mo interacted with $\mathrm{FeCrAl}$ and slightly corroded due to the cracks formed in the outer coating. This can be observed in Fig. 18. The darker regions are visible where the $\mathrm{FeCrAl}$ was thinner (top of the tube) and where the inner load was the highest (lower third of the pressure tube).

\section{Discussion and Conclusions}

The coated cladding is a very promising candidate as an Accident Tolerant Fuel material. It can be developed in a reasonably short time and it is similar to the current fuel system which will result in easier licensing and less challenging adoption of the technology by the industry. However, there are still many unresolved issues related to the material behavior in-reactor. This includes coated cladding creep and periodic strains that occur during normal operation. An experimen- 


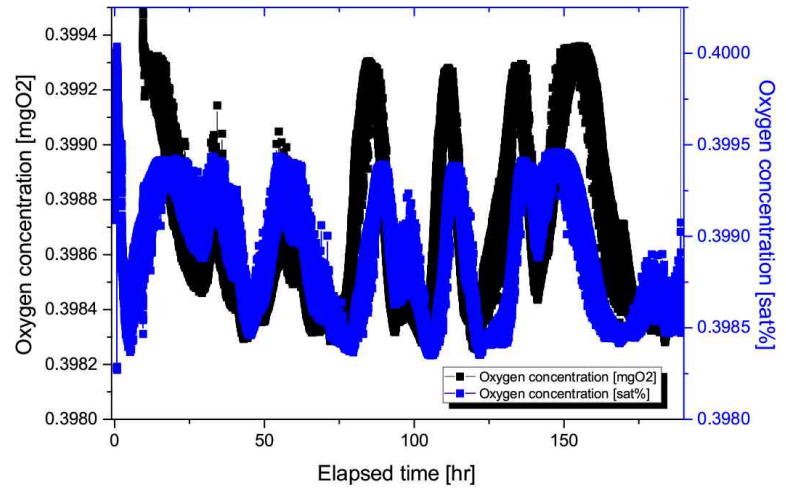

Figure 17. FeCrAl+Mo Run 3 - oxygen concentration.

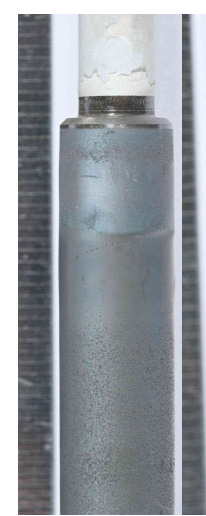

(A) . Top

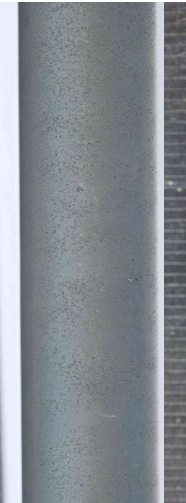

(B) . Overcoat

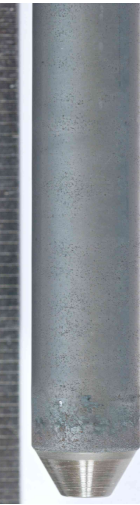

(C)

Bot-

tom
Figure 18. Visual inspection of $\mathrm{FeCrAl}+\mathrm{Mo}$ pressure tubes after Run 1, Run 2 and Run 3 - tension.

tal system was designed and build to test thermal creep of these materials out-of-pile with consequent corrosion of the material in HWC environment. The cladding first creeps down as a result of differential pressure and then the pellet-cladding interaction is simulated using high Ar pressure that leads also to outward creep.

Five different materials were studied (Zry-4, $\mathrm{Zry}+\mathrm{FeCrAl}, \mathrm{Zry}+\mathrm{Mo}, \mathrm{Zry}+\mathrm{Cr}, \mathrm{Zry}+\mathrm{Mo}+\mathrm{FeCrAl})$ and results of the last multicomponent concept presented. There is a mismatch between fundamental physical parameters between each of the coatings and substrate (e.g. thermal expansion, creep rate, swelling). The differences will lead to high stresses in the material and strains during the long-term operation and they can even jeopardize cladding integrity. As shown in Fig. 19 the strains of the cladding material lead to cracking of the coating layers. It should be noted that the thicknesses of the studied materials are higher than expected for the final concepts, therefore, the related stresses are higher as well. For example, the $\mathrm{FeCrAl}+\mathrm{Mo}$ cladding includes $25 \mu \mathrm{m}$ Mo layer with about $150 \mu \mathrm{m}$ layer of $\mathrm{FeCrAl}$ as shown in Fig. 20. The multicomponent cladding with two coating layers brings more challenges due to more interacting layers.

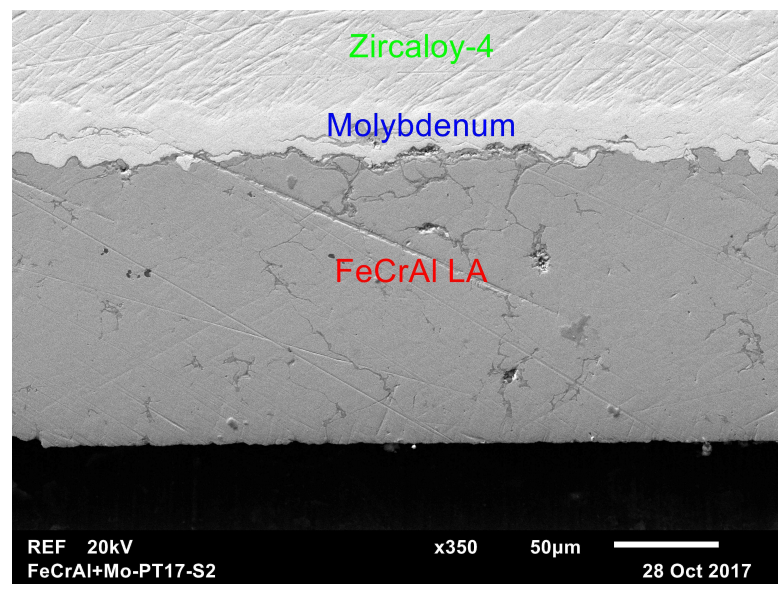

Figure 19. SEM micrograph of the PT17 sample after the test (all three runs). The cracks in both $\mathrm{FeCrAl}$ and Mo coating are visible. There is no spallation visible but the bonding between $\mathrm{FeCrAl}$ and $\mathrm{Mo}$ was reduced.

The SEM micrographs show that cracks first initiated at the $\mathrm{Mo} / \mathrm{FeCrAl}$ interface. Molybdenum is harder and more brittle than both Zry-4 and FeCrAl. It cannot accommodate as much strain as the other two materials, therefore, the crack initiation starts here.

The results showing inlet and outlet water conductivity during the test are directly related to corrosion of the samples. Unfortunately, it cannot be distinguished which of the samples placed inside the autoclave at the same time caused the observed effects. Separate corrosion tests of Mo-coated samples showed rapid corrosion and volatility of the pure Mo coating in HWC and NWC environment. Based on the testing of pure $\mathrm{Mo}$ and $\mathrm{FeCrAl}$ it can be distinguished when Mo was exposed to the environment. There was an increase at the beginning of the first run which is probably related to the defects in the coating from fabrication. Second increase was observed during the second run when probably most of the cracking in coatings happened. The last increase was after 140 hours of the third run and is related to a single failure in one of the samples.

Energy-dispersive X-ray spectroscopy was performed to study the elemental composition at the cross-section of the samples. The EDS line scan did not confirm the diffusion and mixing of the materials. There are bonding layers at the interfaces between each layer that were challenged by the test where neighboring materials are partially mixed. EDS is however not very sensitive to low concentrations and more detailed study using WDS is needed. The ESD also confirms the composition of each layer and their thickness as shown in Fig. 20.

Coated cladding materials have been widely tested in accidental conditions where they can bring additional benefits. However, to utilize them in commercial reactors they also need to perform well during normal operation. Thermal inward creep and outward 


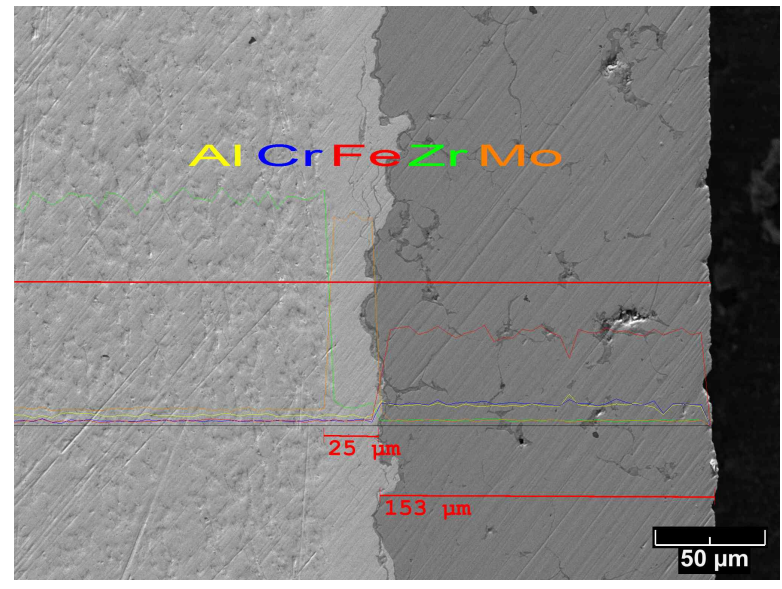

FigURE 20. EDS linescan with SEM micrograph of the PT17 sample. The thickness of the Mo interlayer is around $25 \mu \mathrm{m}$ and FeCrAl LA coating is about 150 $\mu \mathrm{m}$ thick. High number of cracks is visible in both Mo and $\mathrm{FeCrAl}$.

displacement of cladding caused by pellet-cladding interaction are the standard behavior of the Zr-based fuel system. As was shown, these standard conditions might challenge multicomponent cladding mainly due to mismatch of the fundamental physical properties of the applied layers. The tests were performed outof-pile and it is expected that in-pile testing with irradiation creep, swelling or growth will challenge the multicomponent cladding even more.

\section{ACKNOWLEDGEMENTS}

The support for this work was provided by US Department of Energy Integrated Research Project Grant: DENE0008416. Chrome Coated samples were provided by Victor Champagne (ARL), Matt Siopis (UTRC) and Aaron Nardi (UTRC). The FeCrAl and Mo coated samples were coated by IRP team at University of Wisconsin-Madison.

\section{REFERENCES}

[1] J. P. Foster, M. A. McGrath. In-Reactor Creep Behavior of Zircaloy-2. Tech. rep., American Nuclear Society - ANS, La Grange Park (United States), 2007.

[2] K. L. Murty, S. Gollapudi, K. Ramaswamy, et al. 3 Creep deformation of materials in light water reactors (LWRs). In K. L. Murty (ed.), Materials Ageing and Degradation in Light Water Reactors, Woodhead Publishing Series in Energy, pp. 81-148. Woodhead Publishing, 2013. DOI:10.1533/9780857097453.1.81.

[3] H. MAKI, T. HARA. Out-of-Reactor Study on External-Pressure Creep of Zircaloy-2 Fuel Cladding Tubes. Journal of Nuclear Science and Technology 12(1):43-52, 1975. DOI:10.1080/18811248.1975.9733067
[4] B. G. Kim, J. L. Rempe, D. L. Knudson, et al. In-situ Creep Testing Capability Development for Advanced Test Reactor. Tech. Rep. INL/EXT-10-17779, Idaho National Laboratory (INL), 2010. DOI:10.2172/989906.

[5] J. BROCHARD, F. BENTEJAC, N. HOURDEQUIN, et al. Modelling of Pellet Cladding Interaction in PWR fuel p. 8 .

[6] D. R. Olander. Fundamental aspects of nuclear reactor fuel elements: Solutions to problems. Tech. rep., California Univ., Berkeley (USA). Dept. of Nuclear Engineering, 1976.

[7] K. A. Terrani. Accident tolerant fuel cladding development: Promise, status, and challenges. Journal of Nuclear Materials 501:13-30, 2018. DOI:10.1016/j.jnucmat.2017.12.043

[8] M. Ševeček, A. Gurgen, A. Seshadri, et al. Development of Cr cold spray-coated fuel cladding with enhanced accident tolerance. Nuclear Engineering and Technology 50(2):229-236, 2018. DOI:10.1016/j.net.2017.12.011

[9] J. Krejci, M. Sevecek, L. Cvrcek. Development of Chromium and Chromium Nitride Coated Cladding for VVER Reactors. 2017 WRFPM .

[10] C. Tang, M. Stueber, H. J. Seifert, M. Steinbrueck. Protective coatings on zirconium-based alloys as accidenttolerant fuel (ATF) claddings. Corrosion Reviews 35(3):141-165, 2017. DOI:10.1515/corrrev-2017-0010

[11] M. Ševeček, J. Kubáň, M. Valach, R. Škoda. Development of high thermal conductivity UO2-Th heterogeneous fuel. Progress in Nuclear Energy 108:489-496, 2018. DOI:10.1016/j.pnucene.2018.06.012

[12] M. Wagih, B. Spencer, J. Hales, K. Shirvan. Fuel performance of chromium-coated zirconium alloy and silicon carbide accident tolerant fuel claddings. Annals of Nuclear Energy 120:304-318, 2018. DOI:10.1016/j.anucene.2018.06.001

[13] ASTM International, West Conshohocken, PA. ASTM B614-16 Standard Practice for Descaling and Cleaning Zirconium and Zirconium Alloy Surfaces 2016.

[14] B. Maier, H. Yeom, G. Johnson, et al. Development of Cold Spray Coatings for Accident-Tolerant Fuel Cladding in Light Water Reactors. JOM 2017. DOI:10.1007/s11837-017-2643-9.

[15] P. Scott, P. Combrade. Corrosion in pressurized water reactors. Corrosion: Environments and Industries 13 C:362-385, 2006. 